\title{
Do Close-in Giant Planets Orbiting Evolved Stars Prefer Eccentric Orbits?
}

\author{
Samuel K. Grunblatt ${ }^{1}\left(\mathbb{0}\right.$, Daniel Huber ${ }^{1,2,3,4}$, , Eric Gaidos $^{5}$, Eric D. Lopez ${ }^{6}$, Thomas Barclay ${ }^{6}{ }^{(0)}$, Ashley Chontos $^{1}$, \\ Evan Sinukoff $^{1,7}$ (D), Vincent Van Eylen ${ }^{8}$ (D), Andrew W. Howard ${ }^{7}$ (D), and Howard T. Isaacson ${ }^{9}$ (D) \\ ${ }^{1}$ Institute for Astronomy, University of Hawaii, 2680 Woodlawn Drive, Honolulu, HI 96822, USA; skg@ifa.hawaii.edu \\ ${ }^{2}$ Sydney Institute for Astronomy (SIfA), School of Physics, University of Sydney, NSW 2006, Australia \\ ${ }^{3}$ SETI Institute, 189 Bernardo Avenue, Mountain View, CA 94043, USA \\ ${ }^{4}$ Stellar Astrophysics Centre, Department of Physics and Astronomy, Aarhus University, Ny Munkegade 120, DK-8000 Aarhus C, Denmark \\ ${ }^{5}$ Department of Geology \& Geophysics, University of Hawaii at Manoa, Honolulu, HI 96822, USA \\ ${ }^{6}$ NASA Goddard Space Flight Center, Greenbelt, MD 20771, USA \\ ${ }^{7}$ California Institute of Technology, Pasadena, CA 91125, USA \\ ${ }^{8}$ Leiden Observatory, Leiden University, posts 9513, 2300RA Leiden, The Netherlands \\ 9 Department of Astronomy, UC Berkeley, Berkeley, CA 94720, USA \\ Received 2018 May 7; revised 2018 May 29; accepted 2018 June 6; published 2018 June 27
}

\begin{abstract}
The NASA Kepler and K2 Missions have recently revealed a population of transiting giant planets orbiting moderately evolved, low-luminosity red giant branch stars. Here, we present radial velocity (RV) measurements of three of these systems, revealing significantly non-zero orbital eccentricities in each case. Comparing these systems with the known planet population suggests that close-in giant planets around evolved stars tend to have more eccentric orbits than those around main sequence stars. We interpret this as tentative evidence that the orbits of these planets pass through a transient, moderately eccentric phase where they shrink faster than they circularize due to tides raised on evolved host stars. Additional RV measurements of currently known systems, along with new systems discovered by the recently launched NASA Transiting Exoplanet Survey Satellite (TESS) mission, may constrain the timescale and mass dependence of this process.
\end{abstract}

Key words: planets and satellites: dynamical evolution and stability - planets and satellites: gaseous planets planet-star interactions

Supporting material: data behind figure

\section{Introduction}

The NASA Kepler mission has discovered thousands of extrasolar planets, allowing populations of planets orbiting different types of stars to be compared (Howard et al. 2012; Petigura et al. 2013; Dressing \& Charbonneau 2013; Santerne et al. 2016; Fulton et al. 2017; van Sluijs \& Van Eylen 2018). However, the population of planets around evolved stars remained poorly described because so few have been discovered to date, particularly at orbital distances of $0.5 \mathrm{au}$ or less (Sato et al. 2005; Johnson et al. 2010; Lillo-Box et al. 2014; Barclay et al. 2015; Jones et al. 2016).

It has been suggested that the planet population of evolved stars should look quite different from their main sequence counterparts due to dynamical interactions driven by stellar evolution (Veras 2016). Accelerated angular momentum exchange should cause the planet to spiral in to the host star (Zahn 1977; Hut 1981; MacLeod et al. 2018). This results in a scenario where orbital decay happens faster than circularization, producing a population of transient, moderately eccentric close-in planets around evolved stars that are not seen around main sequence stars (Villaver \& Livio 2009; Villaver et al. 2014). The increase in planetary heating from both elevated stellar irradiation and tides raised on the planet will likely also cause inflation of these planets at late times (Bodenheimer et al. 2001; Lopez \& Fortney 2016).

Two well-characterized, close-in inflated giant planets orbiting moderately evolved, or low-luminosity, red giant branch stars, K2-97b and K2-132b, were recently discovered by the $K 2$ extension to the Kepler mission (Grunblatt et al. 2016, 2017). Here, we report new radial velocity (RV) measurements of these planets, as well as RV measurements of a previously validated planet orbiting an evolved star observed by the original Kepler mission, Kepler-643 (Huber et al. 2013; Morton et al. 2016). These measurements allow us to constrain the orbital eccentricities of these planets, which motivates an investigation of the orbital eccentricities of the population of planets around giant stars compared to dwarf stars.

\section{Observations}

RV measurements of K2-97, K2-132, and Kepler-643 were obtained between 2016 January 27 and 2018 February 1 using the High Resolution Echelle Spectrometer (HIRES) on the Keck-I Telescope at the Mauna Kea Observatory in Hawaii. Individual measurements and orbit solutions are shown in Figure 1. All RV spectra were obtained through an iodine gas cell. In order to constrain orbital parameters, we fit the RV data using the publicly available software package RadVel (Fulton et al. 2018). The orbital period of the planets were fixed to published values from transit measurements (Morton et al. 2016; Grunblatt et al. 2017), while we fit for the semiamplitude, phase, and modified eccentricity parameters of the orbit (Eastman et al. 2013). We also fit for an RV jitter term for our measurements and obtained a value between 5 and $10 \mathrm{~m} \mathrm{~s}^{-1}$ for all of the systems studied here. We adopted the same method for determining RVs as described in Butler et al. (1996).

Because RV measurements are not usually taken at regular time intervals, data sampling is often uneven and thus introduces orbital parameter biases, potentially inflating eccentricities beyond their true value (Eastman et al. 2013). 


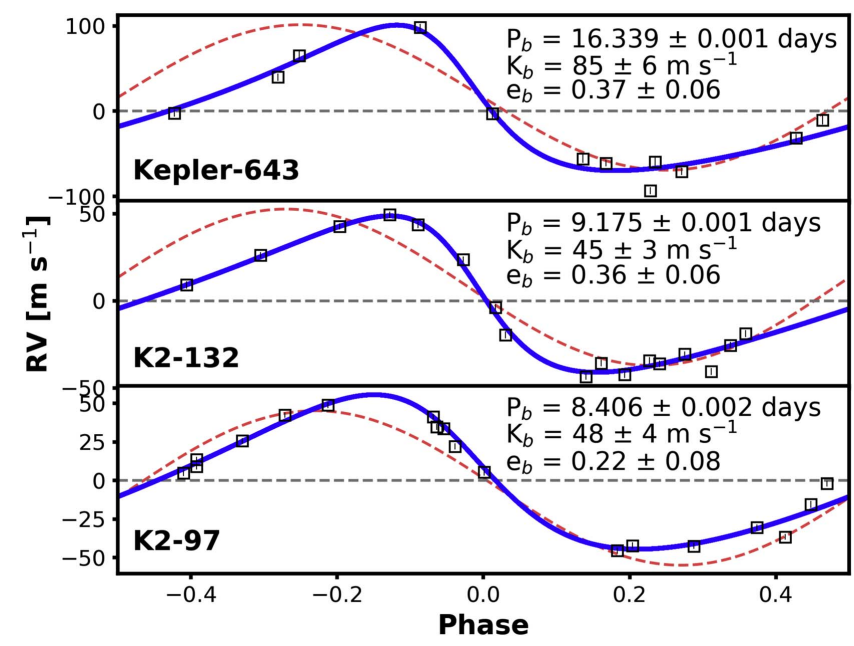

Figure 1. Keck/HIRES radial velocity observations of Kepler-643 (top), K2-132 (center) and K2-97 (bottom), three systems where close-in giant planets orbit evolved stars. All of the orbits display moderate eccentricities between 0.2 and 0.4 . The planets appear to follow a trend, where those on longer orbits are more eccentric than those orbiting their host star more closely. Circular orbits are shown as red dotted lines for reference. (The data used to create this figure are available.)

To ensure that our measured eccentricities are robust, we produced 100 artificial RV data sets of circular orbits for each system, with equivalent orbital periods, semi-amplitudes, and random scatter as measured in our real data, taken at the same times as our real measurements. We then recovered an orbit from each artificial data set using the same techniques given for our real RV data. We find that the distribution of eccentricities recovered from fitting the artificial data sets is consistent with zero in all cases. For all best-fit orbit solutions for the simulated, $e=0$ orbit generated data, we do not recover an eccentricity of greater than 0.1 . We therefore conclude that the eccentricities found by our analysis are not due to sparse sampling of our RV measurements.

\section{Eccentricity Distributions around Evolved Stars}

Figure 2 illustrates the population of known giant planets with published eccentricities orbiting giant stars, as well as the equivalent planet population orbiting dwarfs in the orbital period and eccentricity plane (left) and the $a / R_{*}$ and eccentricity plane (right). Planets are designated as giants if $R_{p}>0.4 R_{\mathrm{J}} .419$ dwarf star systems and 136 giant star systems with constrained eccentricities listed in the NASA Exoplanet Archive are included in our figure (Akeson et al. 2013). Transiting systems are shown as filled circles, while nontransiting systems are shown as empty circles. For nontransiting systems, planet radii were estimated using the massradius relations of Chen \& Kipping (2017). Distinctions as giant or dwarf star systems were made using the physically motivated boundaries in effective temperature and surface gravity described in Huber et al. (2016). Stellar parameters have been taken from the NASA Exoplanet Archive, and individual sources for all known close-in giant planets with published eccentricities orbiting giant stars are listed in Table 1. Our new RV measurements give tentative evidence that the dwarf and giant system eccentricity distributions are inconsistent at periods $\lesssim 50$ days and $a / R_{*} \lesssim 10$.
Figure 3 illustrates the cumulative distributions of eccentricities for various different planetary system samples analyzed here. When considering planets of all sizes, close-in planets show a tendency for low eccentricities. However, this preference is not as strong when considering only giant planets, which is likely due to trends related to planet multiplicity (Van Eylen \& Albrecht 2015; Xie et al. 2016). Remarkably, comparing the population of giant planets orbiting at $\lesssim 50$ day orbital periods, as well as all known planets around giant stars (red lines) to the equivalent planet population orbiting dwarf stars (black lines), illustrates a stronger preference for moderate eccentricities in giant star systems than is seen in dwarf star systems.

To evaluate the significance of the difference between the dwarf and giant star planet populations, we compared the median eccentricities for both populations (see Figures 2 and 3$)$. We restrict our analysis to giant $\left(>0.4 R_{\mathrm{J}}\right)$ planets with orbital periods between 4.5 and 30 days and published eccentricity constraints. This ensures that all of the planets compared here could have been detected around both dwarf and low-luminosity red giant branch stars observed by $K 2$. Furthermore, this sample includes the closest-in known transiting planets orbiting evolved stars while rejecting the shortest period dwarf system planets, which likely would be engulfed by evolved stars due to their large sizes. It also minimizes biases due to planets found in surveys that were particularly well-suited to discovering short-period giant planets on circular orbits around dwarf stars (e.g., WASP, Pollacco et al. 2006). Planets with published upper limits on eccentricity are treated as having circular orbits with error distributions that reach the listed upper limit at a 1- $\sigma$ confidence interval. We find a median eccentricity of $0.152_{-0.042}^{+0.077}$ for close-in giant planets orbiting evolved stars, and a median eccentricity of $0.056_{-0.006}^{+0.022}$ for close-in giant planets orbiting dwarfs.

We also tested the sensitivity of these values to increasing the planet radius cut to $>0.8 R_{\mathrm{J}}$, as well as adjusting the inner period bound between 3 and 8 days, and the outer period bound between 25 and 80 days. We find that our statistics are only significantly affected by changing the inner period bound, driven by the small number of close-in planets known orbiting evolved stars. Thus, we choose bounds to include all of the known close-in planets orbiting evolved stars, while minimizing the number of close-in planets around dwarf stars without an evolved counterpart population.

To further quantify the significance of the eccentricity dichotomy between the populations of giant planets orbiting dwarf and giant stars, we calculate the Anderson-Darling statistic, which is more robust to different-sized and small number distributions than similar tests such as the KolmogorovSmirnov statistic (Simpson 1951; Stephens 1974). We find that both samples are drawn from the same parent population in $6.3 \%$ or fewer of cases. Adjusting our planet radius and period cuts, we find that both samples are drawn from the same parent population in $3.8 \%-15.4 \%$ or fewer of cases for all of the tested samples. This range is dominated by stochastic variation due to the small sample of evolved systems.

As an additional test, we performed a Monte Carlo simulation in which we drew an equal number of eccentricity values from the eccentricity distributions of our bias-resistant sample of close-in giant planets orbiting dwarf stars and giant stars in 4.5-30 days. We find that after repeating this process 

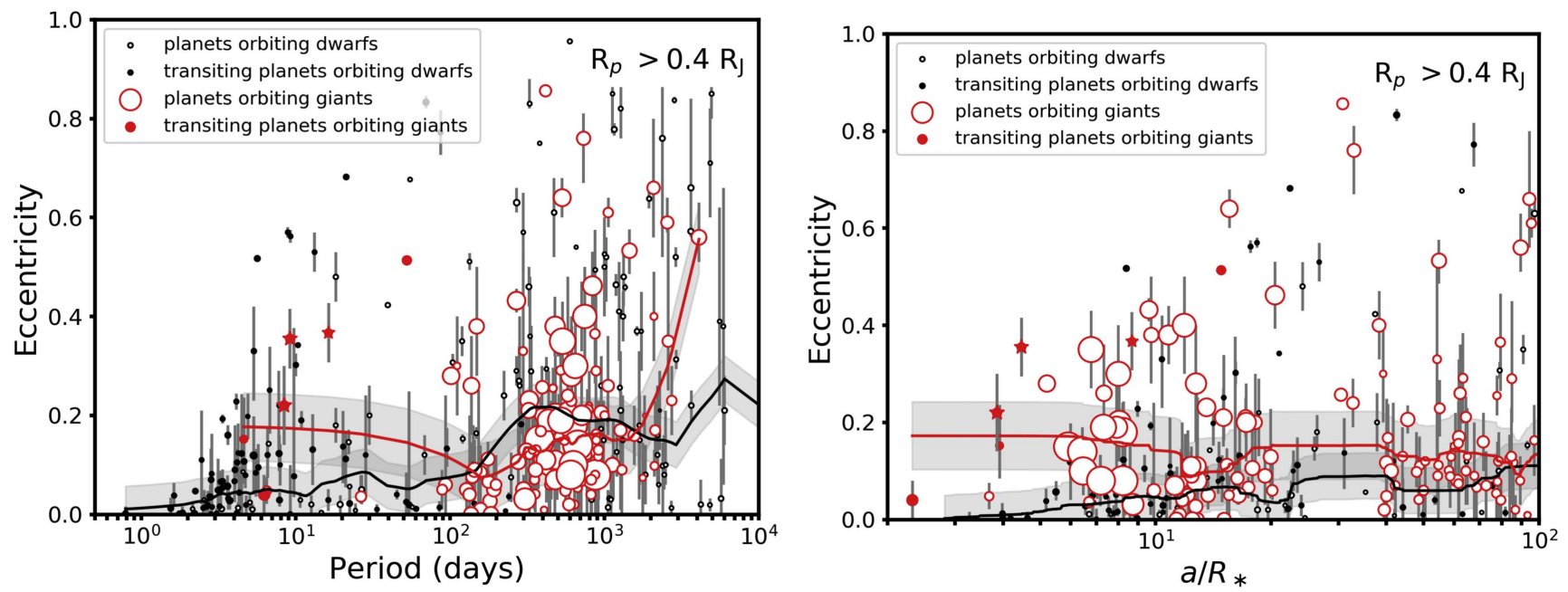

Figure 2. Left: orbital period vs. eccentricity for all giant $\left(>0.4 R_{\mathrm{J}}\right)$ planets with published eccentricities orbiting giant and dwarf stars. Stellar radius scales with the size of the points; planets orbiting giant stars are shown in red, while planets orbiting dwarfs are shown in black. The systems with eccentricities measured in this Letter are highlighted as red stars. A locally weighted regression of the eccentricities are shown by the solid black and red lines for the dwarf and giant star populations, respectively. Right: same as the left, except with $a / R_{*}$ on the $x$-axis.

one million times, the random sample of planets drawn from the dwarf star sample has an equal or higher median eccentricity than the planets orbiting giant stars in $5.7 \%$ of cases, with a range of $4.1 \%-16.7 \%$ for all of the period and radius ranges tested. We also performed the same test for the population of all close-in planets known around dwarf and giant stars, as well as all planets known around dwarf and giant stars, and find that the dwarf star sample has a similar or higher median eccentricity in $0.34 \%$ and $10.6 \%$ of cases, respectively.

Thus, based on our statistical tests, we conclude that closein, evolved star system planets display different eccentricity characteristics than close-in dwarf star system planets at a 1- to $2-\sigma$ level. We note that this is a conservative estimate, as many early literature estimates of eccentricities for both types of systems may be biased toward higher eccentricities due to the mischaracterization of systematic and astrophysical uncertainties (Eastman et al. 2013). More recent RV studies, using newer analysis packages such as RadVel, account for this artificial bias. Re-analysis of RV measurements used to constrain the population of planetary eccentricities could remove this bias, but is beyond the scope of this Letter.

\section{Discussion}

The formation of close-in giant planets is commonly explained by three different hypotheses: in situ formation, disk migration, and tidal migration (see Dawson \& Johnson (2018) for a recent review). Populations of eccentric giant planets are generally viewed as evidence for tidal migration, as they cannot be explained by the other two prevailing mechanisms. Although these planets support tidal migration theory for close-in giant planet formation, we assert that unlike those around dwarf stars, these close-in giant planets are actively undergoing tidal migration, sped up by the late-stage evolution of their host stars. An observed correlation between stellar host evolutionary state and long-period, planetary companions to close-in giant planet systems supports this (Lillo-Box et al. 2016).

Models of the dynamical evolution of close-in giant planets can be strongly affected by the evolution of the host star (Villaver \& Livio 2009; Villaver et al. 2014). The timescale of this dynamical evolution is defined by the tidal interactions between the planet and its host star. Following the reasoning of Villaver et al. (2014), the eccentricity evolution of a planetary orbit will be dominated by planetary tides driving orbit circularization on the main sequence, and stellar tides driving tidal inspiral on the red giant branch. For example, assuming $Q_{p}=Q_{*} \sim 10^{6}$, and using the equilibrium tide formulations of Patra et al. (2017) derived from Goldreich \& Soter (1966), the timescale for orbit circularization for $\mathrm{K} 2-97 \mathrm{~b}$ is $\sim 5 \mathrm{Gyr}$, while the tidal inspiral timescale is $\lesssim 2$ Gyr. This suggests that orbital decay is driven more rapidly than eccentricity evolution as the stellar radius increases, producing a population of transient planets displaying moderate eccentricities at close-in orbits around evolved stars. Though these tidal timescale formulae do not account for planetary or stellar rotation or dynamical tides, these results are consistent with our observations.

Villaver et al. (2014) also predicted that more massive systems evolve more quickly toward lower eccentricities and semimajor axes. This is also tentatively supported by observations, as the most massive hosts in our sample also have the lowest eccentricity orbits (see Table 1). However, a larger sample of systems is needed to confirm this. Correlations between planet and star mass and composition and planetary orbital evolution have not yet been fully explored.

Tidal interaction and migration has long been thought to cause radius inflation in gas-giant planets (Bodenheimer et al. 2001; Storch \& Lai 2014). Increased irradiation due to stellar evolution is also thought to be a source of planetary heating (Lopez \& Fortney 2016). Two of the close-in evolved planets with new RV measurements presented here, K2-97b and K2-132b, show signs of being significantly inflated relative to similar planets seen orbiting main sequence stars (Grunblatt et al. 2017).

To evaluate the dominant radius inflation mechanism for these planets, we follow the prescription for tidal heating given by Miller et al. (2009) and Dobbs-Dixon et al. (2004), and assume the synchronous rotation of the planet and tidal quality factors $Q_{p}=10^{4}$ and $Q_{*}=10^{6}$, within an order of magnitude of observed and model constraints (Gallet et al. 2017; Patra et al. 2017). We find that if the planets are actively circularizing, tidal evolution driven by the star can dominate 
Table 1

Close-in Giant Planets Orbiting Giant Stars

\begin{tabular}{|c|c|c|c|c|c|c|c|}
\hline Name & Mass & Radius & Semimajor Axis & Eccentricity & Stellar Mass & Stellar Radius & Reference \\
\hline $\mathrm{K} 2-132 \mathrm{~b}$ & $0.49 \pm 0.06 M_{\mathrm{J}}$ & $1.30 \pm 0.07 R_{\mathrm{J}}$ & $0.086 \mathrm{au}$ & $0.36 \pm 0.06$ & $1.08 \pm 0.08 M_{\odot}$ & $3.85 \pm 0.13 R_{\odot}$ & 1 , this work \\
\hline K2-97b & $0.48 \pm 0.07 M_{\mathrm{J}}$ & $1.31 \pm 0.11 R_{\mathrm{J}}$ & $0.081 \mathrm{au}$ & $0.22 \pm 0.08$ & $1.16 \pm 0.12 M_{\odot}$ & $4.20 \pm 0.14 R_{\odot}$ & 1 , this work \\
\hline $\mathrm{K} 2-39 \mathrm{~b}$ & $0.125 \pm 0.014 M_{\mathrm{J}}$ & $0.51 \pm 0.06 R_{\mathrm{J}}$ & $0.057 \mathrm{au}$ & $0.15 \pm 0.08$ & $1.19 \pm 0.08 M_{\odot}$ & $2.93 \pm 0.21 R_{\odot}$ & 2 \\
\hline Kepler-91b & $0.81 \pm 0.18 M_{\mathrm{J}}$ & $1.37 \pm 0.07 R_{\mathrm{J}}$ & $0.0731 \mathrm{au}$ & $0.04_{-0.02}^{+0.06}$ & $1.31 \pm 0.1 M_{\odot}$ & $6.30 \pm 0.16 R_{\odot}$ & 5 \\
\hline HD $102956 b$ & $0.96 \pm 0.05 M_{\mathrm{J}}$ & non-transiting & $0.081 \mathrm{au}$ & $0.05 \pm 0.03$ & $1.70 \pm 0.11 M_{\odot}$ & $4.4 \pm 0.1 R_{\odot}$ & 6 \\
\hline TYC3667-1280-1b & $5.4 \pm 0.4 M_{\mathrm{J}}$ & non-transiting & $0.21 \mathrm{au}$ & $0.04_{-0.02}^{+0.04}$ & $1.87 \pm 0.17 M_{\odot}$ & $6.26 \pm 0.86 R_{\odot}$ & 7 \\
\hline
\end{tabular}

References. 1. Grunblatt et al. (2017), 2. Petigura et al. (2017), 3. Huber et al. (2013), 4. Morton et al. (2016), 5. Barclay et al. (2015), 6. Johnson et al. (2010), 7. Niedzielski et al. (2016).

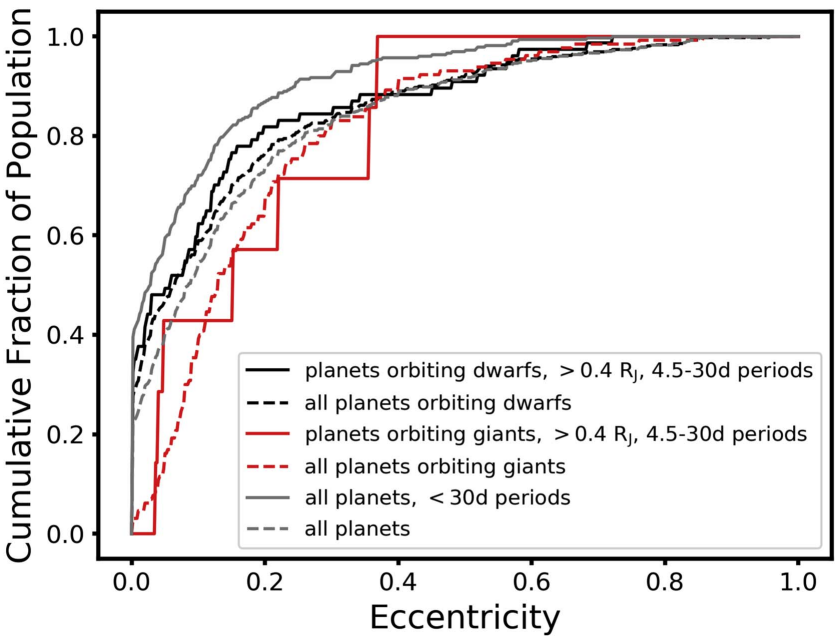

Figure 3. Cumulative eccentricity distributions of different populations of planets. Planets orbiting giant stars (red lines), particularly at periods of 30 days or less, display a preference for moderate eccentricities not seen in dwarf star systems (black lines).

planetary heating by an order of magnitude over irradiative mechanisms. Furthermore, tidal resonance locking may also greatly enhance tidal heating rates (Fuller 2017). Thus, planet radius inflation for these systems may be driven solely by tidal processes.

However, a $Q_{p}$ value of $10^{4}$ and $Q_{*}=10^{6}$ would suggest that the orbit circularization timescale is significantly shorter than the orbital decay timescale. By contrast, the observed eccentricities of these planet orbits suggests that orbit circularization and orbital decay are happening on similar timescales, implying $Q_{*} \sim Q_{p}$. This disagrees with predictions of $Q_{*}$ for evolved stars (Gallet et al. 2017). Furthermore, rotation and/or dynamical tides can drastically change these timescales, and may even increase orbital eccentricity over time (Hut 1981; Fuller 2017). Determining the orbital evolution of evolved systems and causes of late-stage planet inflation will require more in-depth characterization of the combined effect of increased irradiation and tidal energy dissipation on a larger sample of planets.

\section{Summary and Outlook}

The NASA Kepler and $K 2$ missions have recently revealed a population of giant planets at small orbital separations around evolved stars. Here, we report RV observations showing that a majority of these planets display moderate eccentricities, indicating a different evolutionary state for planets around giant stars than those orbiting main sequence stars. This latestage evolution is likely driven by the increase in size of the stellar radius and convective envelope, strongly increasing the angular momentum exchange between the star and the planet, causing the planet to circularize its orbit and spiral into the host star. These two components of orbital evolution must happen on timescales similar enough so that these migrating giant planets with moderate eccentricities appear to be relatively common around evolved stars (Villaver et al. 2014). These planets will thus allow constraints on the determination of the tidal quality factors $Q_{p}$ and $Q_{*}$. Continued follow-up of lowluminosity red giant branch stars will allow estimation of closein planetary occurrence around evolved stars (S. K. Grunblatt et al. 2018, in preparation), which will further constrain our understanding of planetary evolution and dynamical interactions within planetary systems.

Additional eccentricity constraints and more systems are needed in order to confirm the tentative result presented here. The NASA Transiting Exoplanet Survey Satellite (TESS) mission, launched earlier this year, will observe two orders of magnitude as many evolved stars as Kepler and K2, likely resulting in over 100 planet detections around evolved stars (Sullivan et al. 2015; Campante et al. 2016; Barclay et al. 2018). This detection of additional planets orbiting evolved stars will outline the diversity of all such systems, and the likelihood and timescale of planetary system disruption via stellar tides. With this information, we can investigate how quickly planets undergo orbital evolution around low-luminosity red giant branch stars, and at what point planets can no longer survive around giant stars, significantly distinguishing these systems from planet populations of main sequence stars.

The authors would like to thank John Livingston, Jim Fuller, Masanobu Kunitomo, Alessandro Brani, Amaury Triaud, Benjamin Pope, Lauren Weiss, Teruyuki Hirano, Travis Berger, Jessica Stasik, Connor Auge, Aaron Do, and Nader Haghighipour for helpful discussions. This research was supported by the NASA K2 Guest Observer Awards NNX16AH45G and NNX17AF76G to D.H. D.H. acknowledges support by the National Aeronautics and Space Administration under Grant NNX14AB92G issued through the Kepler Participating Scientist Program. This research has made use of the Exoplanet Orbit Database and the Exoplanet Data Explorer at Exoplanets.org. This work was based on observations at the W. M. Keck Observatory granted by the University of Hawaii, the University of California, and the California Institute of Technology. We thank the observers who contributed to the measurements reported here and acknowledge the efforts of the 
Keck Observatory staff. We extend special thanks to those of Hawaiian ancestry on whose sacred mountain of Mauna Kea we are privileged to be guests.

\section{ORCID iDs}

Samuel K. Grunblatt (i) https://orcid.org/0000-0003-4976-9980

Daniel Huber (i) https://orcid.org/0000-0001-8832-4488

Thomas Barclay (ib https://orcid.org/0000-0001-7139-2724

Evan Sinukoff (1) https://orcid.org/0000-0002-5658-0601

Vincent Van Eylen (i) https://orcid.org/0000-0001-5542-8870

Andrew W. Howard (ㄱ) https://orcid.org/0000-0001-

8638-0320

Howard T. Isaacson (10 https://orcid.org/0000-0002-0531-1073

\section{References}

Akeson, R. L., Chen, X., Ciardi, D., et al. 2013, PASP, 125, 989

Barclay, T., Endl, M., Huber, D., et al. 2015, ApJ, 800, 46

Barclay, T., Pepper, J., \& Quintana, E. V. 2018, arXiv:1804:05050

Bodenheimer, P., Lin, D. N. C., \& Mardling, R. A. 2001, ApJ, 548, 466

Butler, R. P., Marcy, G. W., Williams, E., et al. 1996, PASP, 108, 500

Campante, T. L., Schofield, M., Kuszlewicz, J. S., et al. 2016, ApJ, 830, 138

Chen, J., \& Kipping, D. 2017, ApJ, 834, 17

Dawson, R. I., \& Johnson, J. A. 2018, arXiv:1801.06117

Dobbs-Dixon, I., Lin, D. N. C., \& Mardling, R. A. 2004, ApJ, 610, 464

Dressing, C. D., \& Charbonneau, D. 2013, ApJ, 767, 95

Eastman, J., Gaudi, B. S., \& Agol, E. 2013, PASP, 125, 83

Fuller, J. 2017, MNRAS, 472, 1538

Fulton, B. J., Petigura, E. A., Blunt, S., \& Sinukoff, E. 2018, arXiv:1801.01947

Fulton, B. J., Petigura, E. A., Howard, A. W., et al. 2017, arXiv:1703.10375

Gallet, F., Bolmont, E., Mathis, S., Charbonnel, C., \& Amard, L. 2017, arXiv: 1705.10164
Goldreich, P., \& Soter, S. 1966, Icar, 5, 375

Grunblatt, S. K., Huber, D., Gaidos, E., et al. 2016, AJ, 152, 185

Grunblatt, S. K., Huber, D., Gaidos, E., et al. 2017, AJ, 154, 254

Howard, A. W., Marcy, G. W., Bryson, S. T., et al. 2012, ApJS, 201, 15

Huber, D., Bryson, S. T., Haas, M. R., et al. 2016, ApJS, 224, 2

Huber, D., Chaplin, W. J., Christensen-Dalsgaard, J., et al. 2013, ApJ, 767, 127

Hut, P. 1981, A\&A, 99, 126

Johnson, J. A., Aller, K. M., Howard, A. W., \& Crepp, J. R. 2010, PASP, 122,905

Jones, M. I., Jenkins, J. S., Brahm, R., et al. 2016, A\&A, 590, A38

Kunitomo, M., Ikoma, M., Sato, B., Katsuta, Y., \& Ida, S. 2011, ApJ, 737, 66

Lillo-Box, J., Barrado, D., \& Correia, A. C. M. 2016, A\&A, 589, A124

Lillo-Box, J., Barrado, D., Moya, A., et al. 2014, A\&A, 562, A1090.49

Lopez, E. D., \& Fortney, J. J. 2016, ApJ, 818, 4

MacLeod, M., Cantiello, M., \& Soares-Furtado, M. 2018, ApJL, 853, L1

Miller, N., Fortney, J. J., \& Jackson, B. 2009, ApJ, 702, 1413

Morton, T. D., Bryson, S. T., Coughlin, J. L., et al. 2016, ApJ, 822, 86

Niedzielski, A., Villaver, E., Nowak, G., et al. 2016, A\&A, 589, L1

Patra, K. C., Winn, J. N., Holman, M. J., et al. 2017, arXiv:1703.06582

Petigura, E. A., Howard, A. W., \& Marcy, G. W. 2013, PNAS, 110, 19273

Petigura, E. A., Sinukoff, E., Lopez, E. D., et al. 2017, AJ, 153, 142

Pollacco, D. L., Skillen, I., Collier Cameron, A., et al. 2006, PASP, 118, 1407

Santerne, A., Moutou, C., Tsantaki, M., et al. 2016, A\&A, 587, A64

Sato, B., Kambe, E., Takeda, Y., et al. 2005, PASJ, 57, 97

Simpson, P. B. 1951, The Annals of Mathematical Statistics, 22, 476

Stephens, M. A. 1974, J. Am. Stat. Assoc., 69, 730

Storch, N. I., \& Lai, D. 2014, MNRAS, 438, 1526

Sullivan, P. W., Winn, J. N., Berta-Thompson, Z. K., et al. 2015, ApJ, 809, 77

Van Eylen, V., \& Albrecht, S. 2015, ApJ, 808, 126

Van Eylen, V., Albrecht, S., Gandolfi, D., et al. 2016, arXiv:1605.09180

van Sluijs, L., \& Van Eylen, V. 2018, MNRAS, 474, 4603

Veras, D. 2016, RSOS, 3, 150571

Villaver, E., \& Livio, M. 2009, ApJL, 705, L81

Villaver, E., Livio, M., Mustill, A. J., \& Siess, L. 2014, ApJ, 794, 3

Xie, J.-W., Dong, S., Zhu, Z., et al. 2016, PNAS, 113, 11431

Zahn, J.-P. 1977, A\&A, 57, 383 